\title{
Voxel-based automatic multi-criteria optimization for intensity modulated radiation therapy
}

\author{
Yanhua Mai ${ }^{1}$, Fantu Kong ${ }^{1}$, Yiwei Yang ${ }^{3}$, Linghong Zhou ${ }^{1 *}$, Yongbao $\mathrm{Li}^{2^{*}}$ and Ting Song ${ }^{1 *}$ (D
}

\begin{abstract}
Background: Automatic multi-criteria optimization is necessary for intensity modulated radiation therapy (IMRT) because of low planning efficiency and large plan quality uncertainty in current clinical practice. Most studies focused on imitating dosimetrists' planning procedures to automate this process and ignored the fact that organ-based objective functions typically used in commercial treatment planning systems (such as dose-volume function) usually lead to suboptimal plans. To guarantee the optimum results and to automate this process, we incorporate an improved automation strategy and a voxel-based optimization algorithm to generate a novel automatic multi-criteria optimization framework. We then evaluate it in clinical cases.

Methods: This novel automatic multi-criteria optimization framework incorporates a ranked priority-list based automatic constraints adjustment strategy and an in-house developed voxel-based optimization algorithm. Constraints are sequentially adjusted following a pre-defined priority list. Afterward, a voxel-based fluence map optimization (FMO) with an orientation to the newly updated constraints is launched to find a Pareto optimal solution. Loops of constraints adjustment are repeated until each of them could not be relaxed or tightened. The feasibility of the framework is evaluated with 10 automatic generated gynecology (GYN) cancer IMRT cases by comparing the dosimetric performance with the original.

Results: Plan quality improvement is observed for our automatic multi-criteria optimization method. Comparable DVHs are found for the planning target volume (PTV), but with better organs-at-risk (OAR) dose sparing. Among 13 evaluated dosimetric endpoints, 5 of them show significant improvements in automatically generated plans compared with the original plans. Investigation of improvement tendency during optimization exhibits gradual change as the optimization stage proceeds. An initial voxel-based optimization stage and in-low-priority dosimetric criteria tighten can significantly contribute to the optimization procedure.
\end{abstract}

Conclusions: We have successfully developed an automatic multi-criteria optimization framework that can dramatically reduce the current trial-and-error patterned planning workload while affording an efficient method to assure high plan quality consistency. This optimization framework is expected to greatly facilitate precise radiation therapy because of its advantages of planning efficiency and plan quality improvement.

Keywords: Multi-constraints optimization, Voxel-dependent parameter, Treatment planning, Intensity modulated radiation therapy

\footnotetext{
*Correspondence: smart@smu.edu.cn; liyb1@sysucc.org.cn;

songting0129@163.com

${ }^{1}$ Department of Biomedical Engineering, Southern Medical University,

Guangzhou 510515, Guangdong, China

${ }^{2}$ Department of Radiation Oncology, Sun Yat-Sen University Cancer

Center,State Key Laboratory of Oncology in South China, Collaborative

Innovation Center for Cancer Medicine, Guangzhou 510060, China

Full list of author information is available at the end of the article
}

(c) The Author(s). 2018 Open Access This article is distributed under the terms of the Creative Commons Attribution 4.0 International License (http://creativecommons.org/licenses/by/4.0/), which permits unrestricted use, distribution, and reproduction in any medium, provided you give appropriate credit to the original author(s) and the source, provide a link to the Creative Commons license, and indicate if changes were made. The Creative Commons Public Domain Dedication waiver (http://creativecommons.org/publicdomain/zero/1.0/) applies to the data made available in this article, unless otherwise stated. 


\section{Background}

Intensity-modulated radiation therapy (IMRT) treatment plan optimization is a multi-objective problem that aims to provide dose coverage, homogeneity and conformity to planning target volume (PTV) while sparing organs-at-risk (OARs) [1-4]. From a mathematical perspective, directly solving such multi-objective problems can be challenging, whereas a weighted and summed single-objective optimization is more reliable and has been commonly used in commercial treatment planning systems (TPS) [5]. However, the current commercial TPS needs users to input some optimization parameters, including ideal dosimetric goals and the organ-based weighting factors. These factors represent the importance of specific organs that are difficult to assign before optimization. Therefore, the dosimetrists must manually tune these parameters through a trial-and-error process [6-8], which leads to a time-consuming planning procedure; moreover, the final plan's quality highly depends on dosimetrist's experience [9-11].

Several studies have attempted to automate multi-criteria plan optimization. The methods that use a pre-defined constraint priority list and a particular constraints adjustment mechanism have been studied for years. Wilkens et al. came up with a four-step enhancement procedure for automatic multi-criteria planning and used this procedure to evaluate head and neck cases [12]. The goal of each step is as follows: 1) to obtain homogeneous dose distributions for PTV; 2) to reduce the mean dose for OARs (i.e., parotid glands and the oral cavity); 3) to reduce doses for other normal tissues; and 4) to smoothen the fluence maps. These procedures are performed sequentially, and the fourth step incorporates a non-clinical goal. Jee et al. [13] implemented a hierarchical method called lexicographic ordering (LO), which was applied to prostate and head and neck cases. The goals for optimization are also pre-categorized into four levels of priority based on clinical importance. During optimization, the objectives were handled individually in a pre-defined order and consequently changed into constraints. Different from these works, Breedveld et al. [14] adopted an automatic constraint adjustment strategy based on a pre-defined priority list to generate a plan with all constraints met as well as possible. The strategy also includes four stages. The first stage tries to find an initial solution. The second stage relaxes the constraints when the initial solution from stage one does not satisfy all constraints. The third and fourth stages attempt to tighten all constraints to their maximum extent without higher-priority constraints. Breedveld et al. $[15,16]$ later developed an improved automatic multi-criteria plan optimization model based on lexicographic ordering (LO) and showed a close or better plan quality than original plans from the dosimetrists.
However, most previous studies used an organ-based optimization model that has an incomplete mathematical solution space and usually leads to sub-optimal plan quality $[17,18]$. Compared with the organ-based optimization model, studies have shown that a refined voxel-based optimization model can help to navigate the solution from the partial space to the entire Pareto surface [18]. In that case, generating an optimal treatment plan with voxelbased optimization model is practical. For example, Cotrutz and Xing [19, 20] first refined the traditional organ-based optimization objective to a voxel-based one and showed that the dose-volume histogram(s) (DVH) could be greatly improved when the dose distribution is on a local level or with differential shapes of the regionof-interest (ROI). Moreover, Wu et al. [21] proposed a novel voxel-based model and mathematically proved that the voxel-based optimization method can carefully balance the trade-offs between ROIs. They also found that re-optimizing original plans by adjusting voxel-based weighting factors is equivalent to tuning voxel-based threshold doses.

Considering that a complete planning optimization procedure is the repeated iterations of optimization under appropriate constraints, we combined an automatic constraints adjustment mechanism with a voxel-based plan optimization algorithm to build a novel automatic multicriteria optimization framework. This framework automatically adjusted the dosimetric constraints based on their clinical importance and simultaneously explored a wider solution domain with voxel-based optimization model. Constraints are sequentially adjusted based on a pre-defined constraint priority list, and a voxel-based fluence map optimization (FMO) engine with an orientation to the newly updated constraints is then launched to search a Pareto optimal solution. The feasibility of the framework was evaluated with 10 clinically collected gynecology (GYN) cancer IMRT cases by comparing the plans generated by the proposed method with the original clinical ones on plan quality, in terms of DVH curves and their detailed dosimetric endpoints. Furthermore, a paired t-test is performed on detailed dosimetric endpoints to reveal the significant statistical difference between evaluated plans. The transition tendency within the optimization procedures was recorded for further investigation.

\section{Methods}

\section{Overall framework}

The proposed automatic multi-criteria optimization framework includes two main loops (Fig. 1), as follows: an outer loop (blue box), to prospect the most appropriate constraints; and an inner loop (black box), to explore the global optimum. The outer loop first adjusts the constraints automatically based on the optimization results from a previous iteration. It relaxes constraints if they are not satisfied, but 


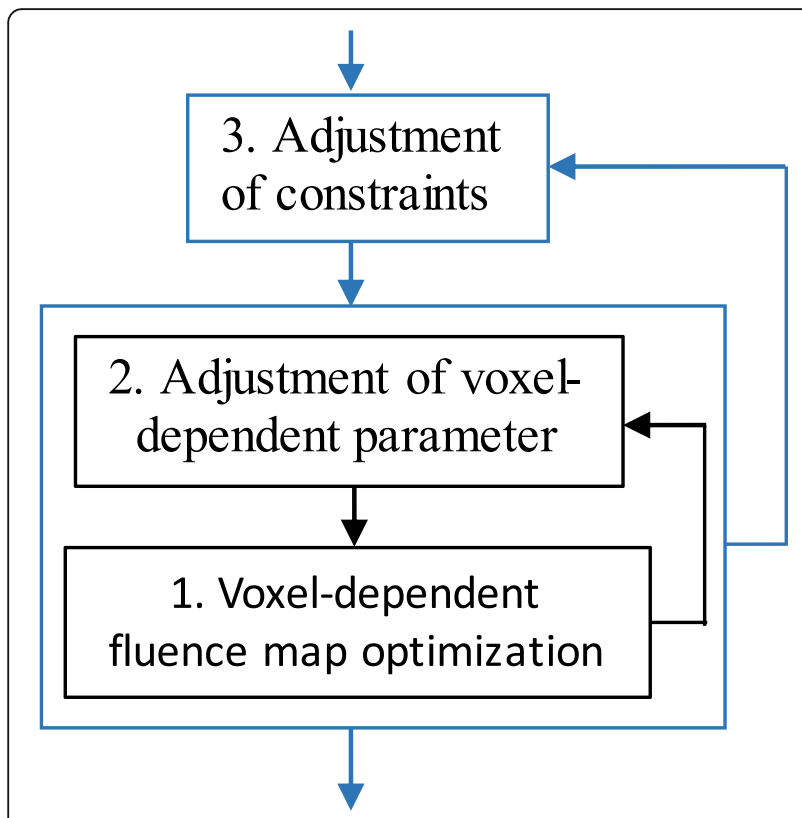

Fig. 1 Proposed automatic multi-constraints optimization framework

otherwise tightens them. Whenever the constraints are set, the voxel-based FMO is immediately launched to obtain a Pareto optimal solution under these constraints. The loop is repeated until all constraints cannot be relaxed or tightened further. The inner loop is the mentioned voxel-based FMO. The loop iterates between two parts. One is the automatic adjustment of voxel-based parameters, and the other is the solver of FMO problem for given voxel-based parameters. Loops will stop when all given constraints in the outer loop are met or the iteration reaches its maximum number. Details are stated carefully in the following parts.

\section{Voxel-based fluence map optimization}

Normally, for IMRT optimization, the primary step is to obtain optimal intensity maps for beams that penetrate from assuming known and fixed angles. Here, we implement a voxel-based quadratic model formula below for fluence map optimization [22].

$$
\begin{array}{r}
s(f)=\sum_{v \in V} \xi_{v}\left(H f-d_{v}^{p}\right)^{T} \tilde{w}_{v}\left(H f-d_{v}^{p}\right)+\kappa(M f)^{T}(M f) \\
\text { s.t. } f \geq 0, D_{x \%} \geq(\leq) d_{1}, V_{x} \leq v_{1}
\end{array}
$$

Notation $f$ is the beamlet intensity vector with all nonzero elements. $H$ is the dose deposition coefficient matrix pre-calculated using the quadrant infinite beam (QIB) [23, 24] algorithm implemented in a computational environment for radiotherapy research (CERR) platform [25]. The dose distribution $d$ is linear to beamlet intensity, and the calculation can be quickly performed by $d=H f$ during optimization. $s(f)$ denotes the objective function of FMO problem, including two terms. The former term is the dose difference between the received dose $H f$ and the reference dose $d_{v}^{p}$ (prescription dose for target, a reasonable low dose for OAR). The latter term is a regularization term that ensures a smooth fluence map. $\kappa$ is the fluence map smooth weighting factor. $M$ is an operator equal to $\Delta f / f$, $\Delta f$ is the discrete Laplacian operator to fluence. Two types of voxel related parameters are incorporated in the former part of Eq. (1), namely, the regular organ-based weight $\xi_{v}$ and the refined voxel-dependent parameter $w_{j}$, which is the element of diagonal matrix $\tilde{w}_{v}$ with its dimension equals to the number of voxels selected in optimization, $j . j \in v$ is the voxel index belongs to ROI $v$. $\xi_{v}$ is the weight assigned for different ROIs $v \in V . D_{x \%} \geq$ $(\leq) d_{1}$ and $V_{x} \leq v_{1}$ are the commonly used dose-volume constraints. When all parameters in Eq. (1) are set, a bound constrained convex quadratic problems (BOXCQP) algorithm [26] is called to solve the optimization problem, thereby achieving a feasible solution in rapid convergence.

\section{Adjustment of voxel-based parameters}

When sets of constraints were updated in the outer loop of the framework, voxel-based parameters should be tuned consequently to satisfy these constraints. Considering that the voxels involved in optimization are enormous, typically $\sim 10^{7}$, an automatic voxel-based parameter adjustment method should therefore be preferred over traditional manual tuning. The principle of adjusting voxel-based parameter is intuitive. We find the voxel-violated constraints and increase their corresponding values.

\section{Penalized voxel selection}

Two main types of constraints are considered in this study, namely, dose and dose-volume constraints. These constraints are commonly used in clinics. Detailed selections for these two situations are shown in Fig. 2. The shaded area is the selected voxel region.

Figure 2 (1) (2) show the selection when constraints are a maximum dose (for PTV and OARs) and minimum dose (for PTV), respectively. $D_{c}$ is the critical dose level. For maximum dose constraints, voxels with received dose $\geq D_{c}$ are selected and their assigned parameters are increased. Similarly, for minimum dose constraints, voxels with received dose $\leq D_{c}$ are selected.

For dose-volume constraints, reducing or increasing doses for voxels with their received dose just over or under the critical dose level is more efficient [27]. Figure 2 (3) (4) show the selection of voxels for maximum dose-volume constraints (for PTV and OARs) and minimum dosevolume constraints (for PTV), $D_{c}$ is the critical dose level, $V_{c}$ is the critical volume, $D$ is the dose level with a volume equals to $V_{c}$. For the maximum dose-volume constraint, 

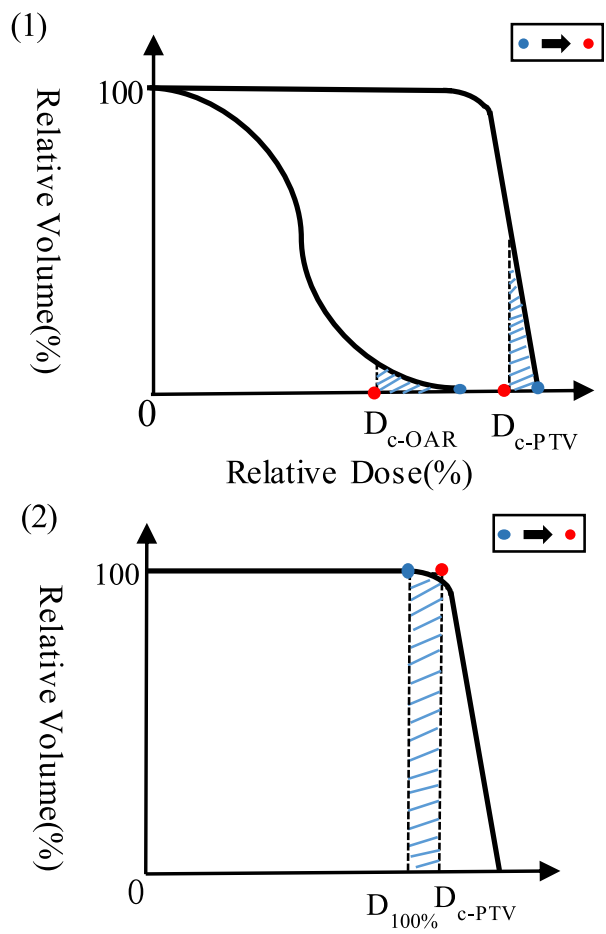

Relative Dose $(\%)$
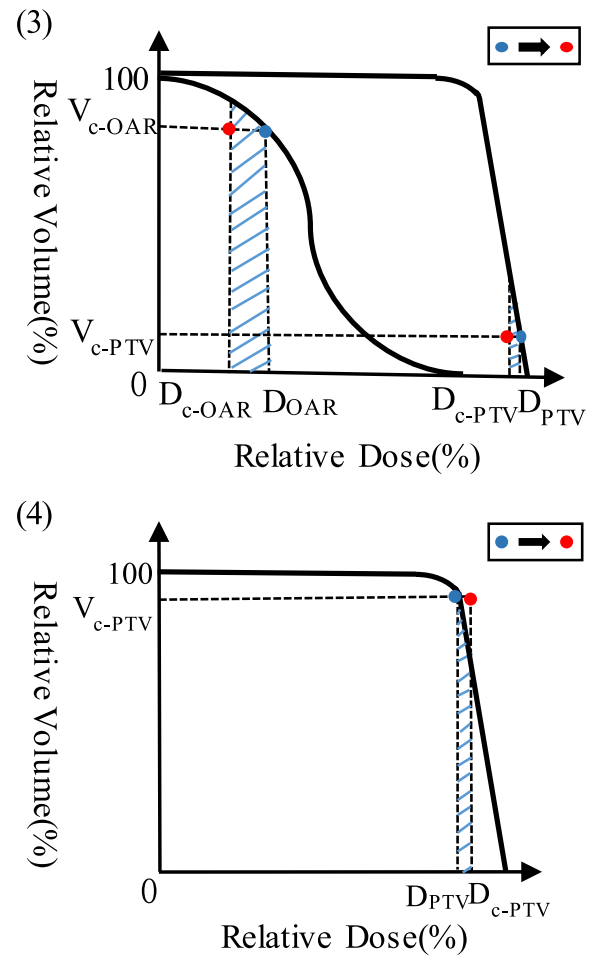

- Current constraint value

- To be satisfied constraint value
Fig. 2 Violation region (shaded area) illustration for PTV and OARs with different constraints. (1) (2) ROls with constraints of maximumdose and minimum-dose; (3) (4) ROls with constraints of maximum and minimum dose-volume

the voxels with dose value between $D_{c}$ and $D\left(D>D_{c}\right)$ are selected. For the minimum dose-volume constraint, the voxels with dose values between $D$ and $D_{c}\left(D<D_{c}\right)$ are selected.

\section{Voxel-based parameter adjustment}

After the selection of penalized voxels, their corresponding parameters are increased accordingly. For minimum dose and dose-volume constraints, voxel-based parameters are updated by $w_{j}^{k+1}=w_{j}^{k}\left[\left(D_{c}+\alpha\right) / D_{j}^{k}\right]^{\mu},\left(D_{j}^{k} \leq D_{c}\right)$. For maximum dose and dose-volume constraints, voxel-based parameters are updated by $w_{j}^{k+1}=w_{j}^{k}$ $\left[\left(D_{j}^{k}+\alpha\right) / D_{c}\right]^{\mu},\left(D_{j}^{k} \geq D_{c}\right)$, where $D_{j}^{k}$ is the received dose for voxel $j$ at $k$ iteration. $w_{j}^{k}$ is the voxel-based parameter for voxel $j$ at previous iteration $k \cdot w_{j}^{k+1}$ is the updated voxel-based parameter for voxel $j$ at current iteration $k+1$. Parameters $\mu$ and $\alpha(\alpha>0)$ are the user-defined enhancement factors. Their effect on the convergence speed are discussed in this paper.

Normally, the optimized dose distribution will meet or become close to the constraints after several iterations of voxel-based parameter update and FMO optimization. When all constraints are met, the iteration is immediately terminated. However, in certain cases, the constraints may be set too tight for this particular patient and are thus hard to meet. Then, the algorithm must force itself to stop by a reasonable maximum iteration time.

\section{Adjustment of constraints}

The promising solution to an efficient and high-quality plan generation is to feed the optimization engine with appropriate constraints based on clinical planning experience. When the given constraints are too tight, the optimization engine cannot easily find a satisfying solution. By contrast, if the constraints are too loose, the achieved plan could be sub-optimal. This situation is the uppermost exact reason for the current trial-and-error planning procedure until the dosimetrists set constraints that are sufficiently tight. Therefore, attempting to automatically adjust these constraints should be addressed (the outer loop in Fig. 1). Prior to the automatic constraints adjustments, a user-defined constraint priority list with some initial values should be given first.

\section{Constraint priority list}

Constraint priority list is a pivotal conversion from the clinical bias to the scientific trade-offs. The list is 
generated by classifying and sequencing the planning endpoints (normally from clinically adopted protocols) following their clinical importance. Endpoints with their constraints are arranged at different levels, with a corresponding number that indicates its priority. Moreover, constraints are divided into two classes, namely, hard and soft constraints. With a priority number 0 , hard constraints cannot be violated and are forbidden to relax or tighten during optimization. By contrast, soft constraints have nonzero priority numbers, and their priorities are decreased gradually with their increasing number. Soft constraints can be relaxed and tightened, and they may be promoted to hard constraints during optimization.

Given an example of priority list set on patients who received IMRT with GYN cancer (Table 1), the optimization requiring dosimetric endpoints and constraints are accordingly derived from a clinical protocol, i.e. the International of Radiotherapy Technology Effectiveness in Cervical Cancer ("INTERTECC") in this study. Among these, the first imperative criteria would be given to $D_{\min }$ (the minimum dose), $D_{\max }$ (the maximum dose), $D_{97 \%}$ (the minimum percentage dose of 97\%) of the PTV, and $D_{\max }$ of the Body- $(\mathrm{PTV}+1)(1 \mathrm{~cm})$ for considering the PTV coverage and dose homogeneity and basic normal tissue protection. Thus, they are set as hard constraints with the "highest" priority number of 0 . Here "Body- $(\mathrm{PTV}+1)$ " is considered rather than "Body-PTV," because a dose transition region between high-dose PTV and low dose normal tissue is usually needed to make the optimization problem easier. Other planning-related OAR endpoints are subsequently considered soft constraints, such as endpoints of rectum, bladder, bowel, femoral heads, and bone. Their priorities are co-determined based on both planning experience and physician's preference. Details are listed as in Table 1.

Table 1 Initial constraint priority list for case 1

\begin{tabular}{llll}
\hline ROls & Endpoints & Initial constraints & Priorities \\
\hline PTV & $D_{\min }$ & $41 \mathrm{~Gy}$ & 0 \\
PTV & $D_{\max }$ & $50 \mathrm{~Gy}$ & 0 \\
PTV & $D_{97 \%}$ & $43.8 \mathrm{~Gy}$ & 0 \\
Body & $D_{\max }$ & $50 \mathrm{~Gy}$ & 0 \\
Body-(PTV + 1) & $D_{\max }$ & $43 \mathrm{~Gy}$ & 0 \\
Rectum & $V_{40}$ & $80 \%$ & 1 \\
Bladder & $V_{40}$ & $65 \%$ & 1 \\
Rectum & $V_{30}$ & $97 \%$ & 2 \\
Bowel & $V_{40}$ & $30 \%$ & 2 \\
Femoral heads & $V_{30}$ & $15 \%$ & 2 \\
Bone & $V_{10}$ & $80 \%$ & 3 \\
Bone & $V_{20}$ & $66 \%$ & 3 \\
\hline
\end{tabular}

\section{Adjustment strategy}

The adjustment of constraints is a four-stage process that has been discussed thoroughly in Breedveld et al. [27]. The first is the preparatory stage. In this stage, we generate an initial dose distribution by applying an in-house developed voxel-dependent parameter optimization. The initial organ-dependent parameters, voxel-dependent parameter (all start from 1), and priority list are the preliminary inputs and are set empirically. Similar to clinical circumstances, constraints set in this stage are consistently too tight. Thus, a solution that can satisfy all the constraints cannot easily be obtained. In this case, partial constraints must be relaxed. This situation leads to the second stage, with an attempt to relax the constraints until each of them can be satisfied. The relaxation begins from low priority to high priority, cluster by cluster. For example, if one or more soft constraints are violated, the lowest priority ones are simultaneously selected for relaxation. A simple but effective means for relaxation is to increase current constraint values with an appropriate interval, such as $0.5 \%$ for most cases based on experience. Results generated in this stage have a high possibility to improve. Thus, in the following third and fourth stages, the potential loose constraints should be tightened until the improvement of any single constraint is accompanied by at least one other constraint violation. By contrast, in the tightening round, constraints are adjusted from high-priority to low-priority, endpoint by endpoint. Each stage calls the automated voxel-dependent FMO with a maximum number of iterations. The soft constraints relaxed in the second stage are tightened in the third stage, and the remaining soft constraints are tightened in the fourth stage. If all constraints are met, including the tightened constraints, the optimization system would continue to tighten the remaining ones until it reaches the maximum iteration. Otherwise, the ongoing constraint is reset to its previous value and becomes promoted as a hard constraint. Similarly, a constraint is tightened by subtracting an appropriate interval such as $0.5 \%$.

\section{Evaluation}

The feasibility and efficiency of this proposed automatic multi-criteria optimization framework are evaluated by 10 GYN IMRT cases, with a prescription dose of 45 Gy to the PTV and treated with seven beams $\left(150^{\circ}, 100^{\circ}\right.$, $50^{\circ}, 0^{\circ}, 310^{\circ}, 260^{\circ}$, and $210^{\circ}$ ). All these IMRT plans are originally exported from the commercial Eclipse TPS (Varian Medical Systems, Palo Alto, CA). We reoptimized each IMRT plan using this in-house developed automatic multi-criteria optimization framework (called an optimized plan below) and compared it with the original one (called the original plan) in terms of 
DVH curves and particular endpoints. Beam set-ups are maintained unchanged.

To investigate synoptically the plan quality improvement, a paired t-test was performed on each plan dosimetric endpoint for all evaluated cases between the optimized plan and the original plan. Furthermore, we recorded the stage-status to observe the transition tendency along with the four-stage optimization proceeded by examining their constraint-settings and subsequent optimized values for each dosimetric endpoint.

Other parameters were set by experience, as follows: organ-based weights $100,1,2,0.1,0.1,0.1,0.1$ and 0.1

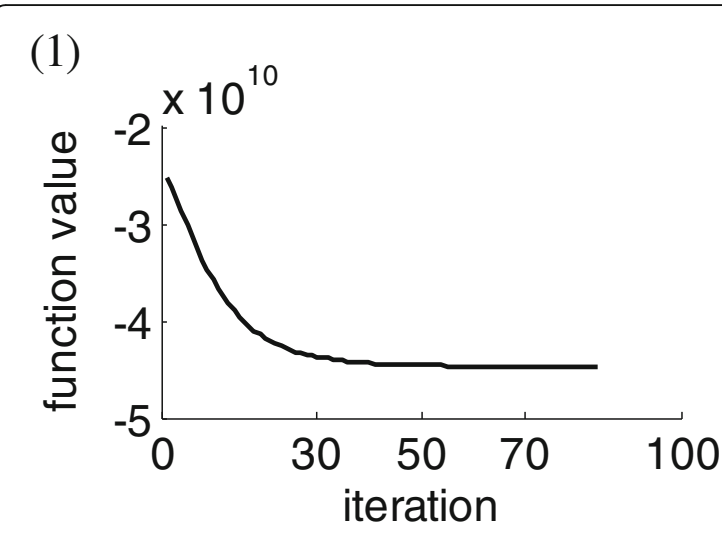

(2)

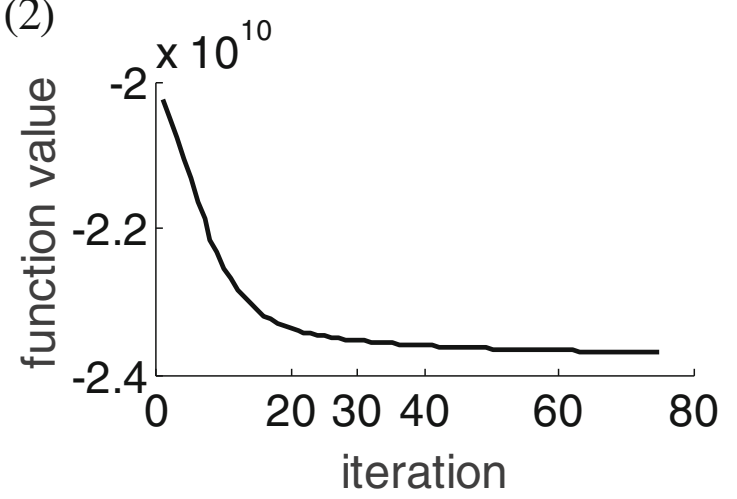

(3)

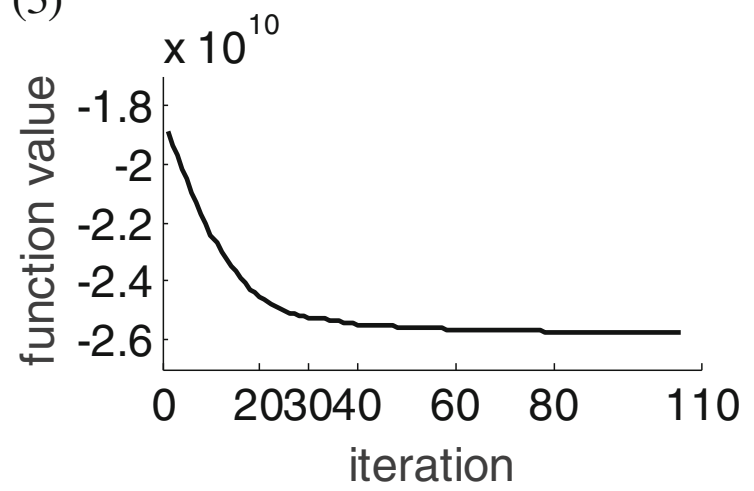

Fig. 3 Convergence speed as with voxel-dependent FMO iterations for three cases for PTV, bladder, rectum, bowel, femoral heads, bone, Body- $(\mathrm{PTV}+1)$, and body, respectively. The initial voxel-based weight was set to 1 , and the initial constraint priority list is shown in Table 1 . The reference dose $d_{v}^{p}$ in Eq. (1) was set to a prescription dose for PTV and empirically for each OAR. Maximum voxel-based parameter updating time was set to $30,10,30$, and 30 for each stage. Here, 30 is determined based on the convergence of the optimization cost function value as with voxel-weight updating time (Fig. 3). Voxel-dependent parameter auto-tuning influence factors $\mu$ and $\alpha$ were 20 and 0.5 , based on the investigation to the convergence when $\mu$ and $\alpha$ ranged from 5 to 25 and $0.001-1$, respectively, based on experience (as shown in Fig. 4). Several of these cases couldn't generate a solution when $\mu$ was during 20 to 25 owing to an ill-condition Hessian matrix. The same situation occurred when $\mu$ was equal to 20 and when $\alpha$ changed from 0.5 to 1. This whole framework was implemented with Matlab 2013a and installed on a $3.4 \mathrm{GHz}$ Intel Core4 computer running Windows 7.
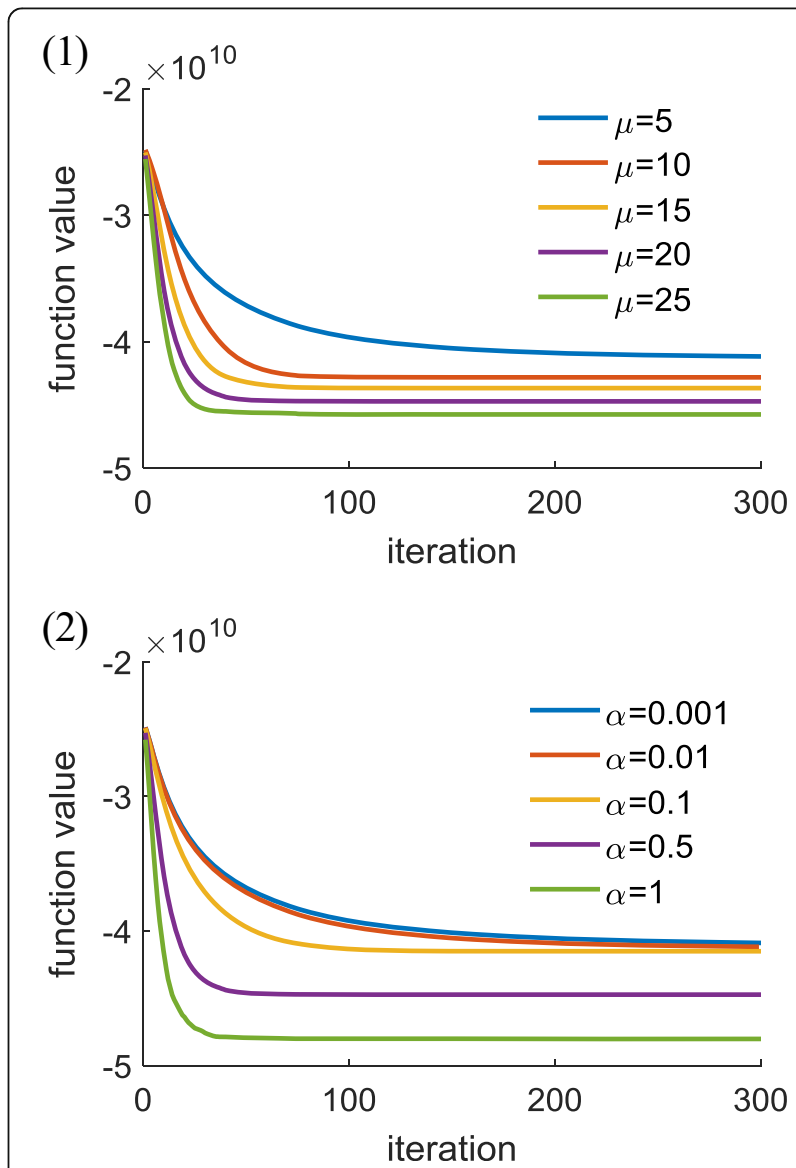

Fig. 4 Convergence speed as with various combination of $\mu$ and $a$. (1): $a=0.5, \mu=5,10,15,20,25 ;(2): \mu=20, a=0.001,0.01,0.1,0.5,1$ 
(1)

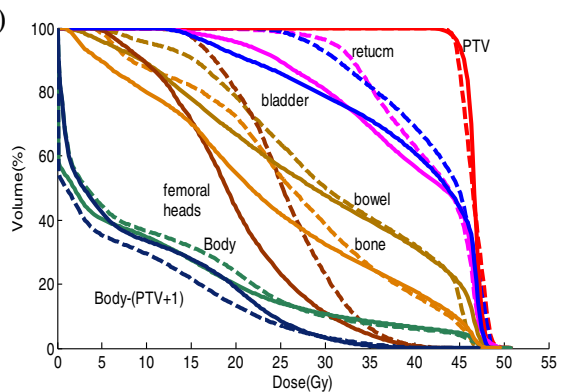

(3)

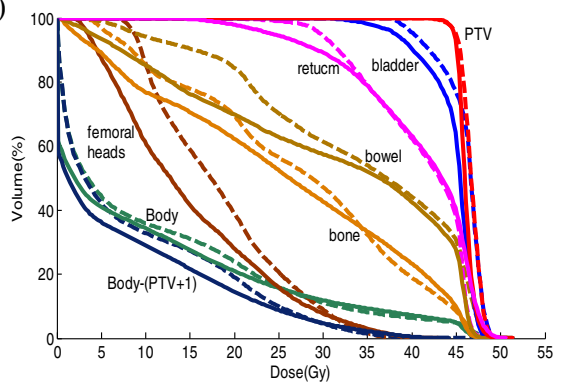

(5)

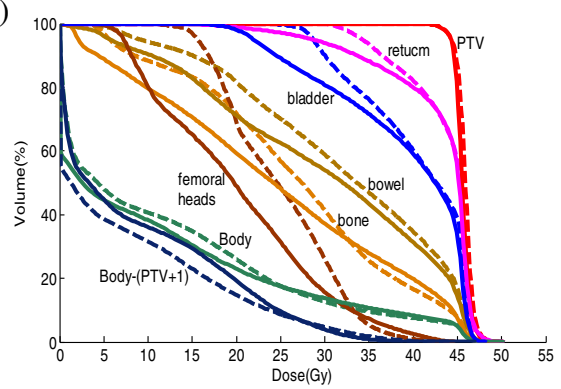

(7)

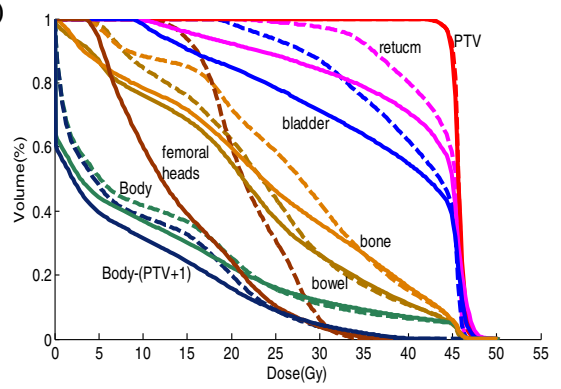

(9)

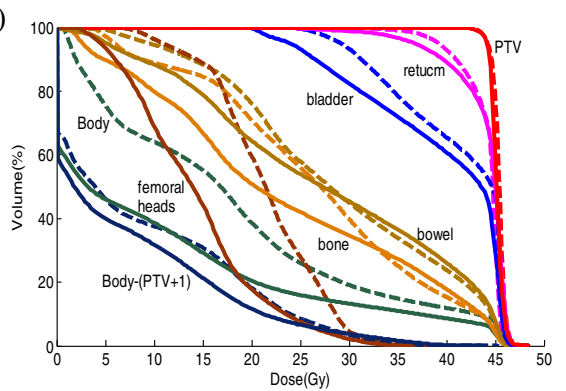

(2)

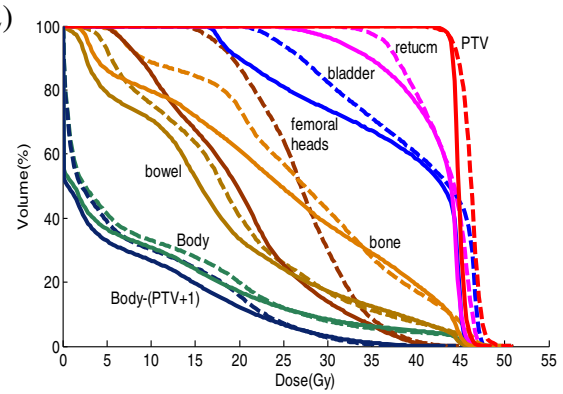

(4)

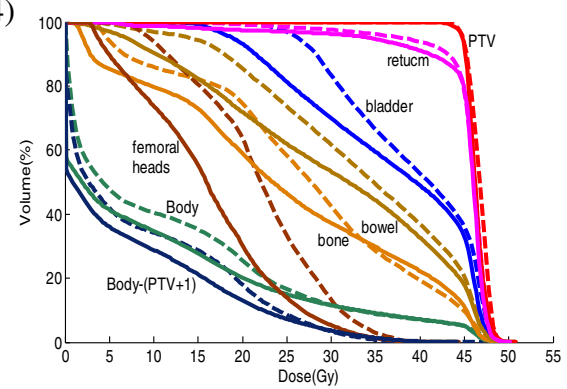

(6)

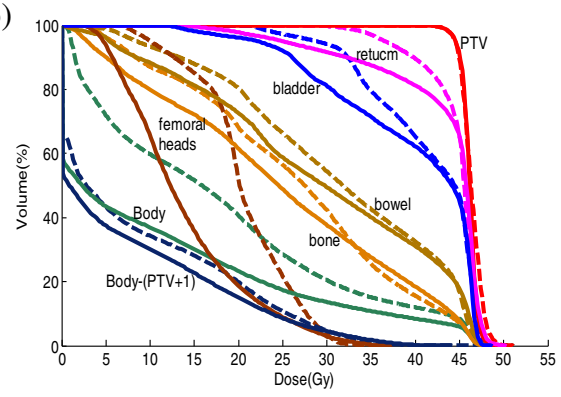

(8)

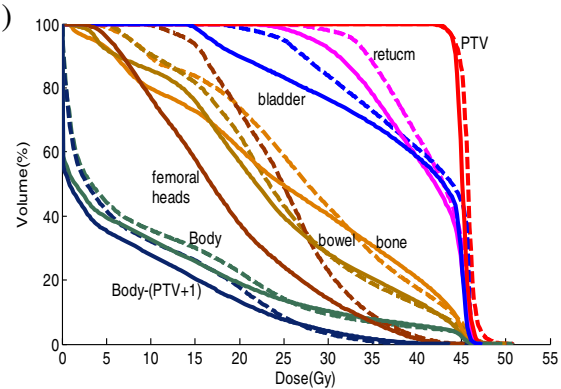

(10)

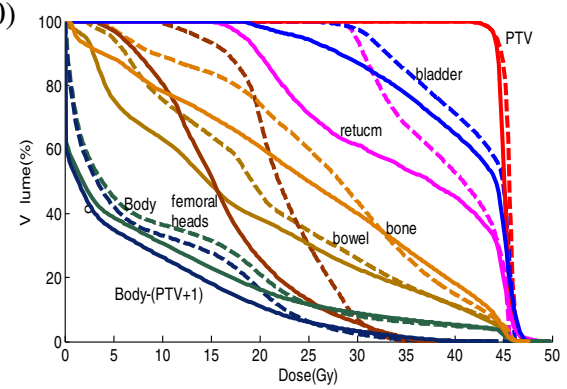

Fig. 5 DVH comparisons of the optimized plan and the original for 10 GYN IMRT cases. Solid line: the optimized plan; Dashed line: the original plan 


\section{Results}

Plan quality improvement

Both DVH curves and particular endpoint comparisons show plan quality improvement for our optimized plans. The DVH comparison of the optimized plans and the original plans for the evaluated 10 GYN IMRT cases is shown in Fig. 5. The optimized plans not only improved the PTV dose homogeneity (with decreased PTV hotspot) but also provided a better OAR dose sparing for most cases. By contrast, Table 2 further illustrates specific DVH endpoint comparison for case 2 as an example. Almost all the endpoints in both plans satisfy the protocol well, except for one soft constraint, i.e., $\mathrm{V}_{30}$ of the rectum, which was violated in both plans because of the large overlapping area with PTV. The other two soft constraints, namely, $\mathrm{V}_{10}$ and $\mathrm{V}_{20}$ of bone, also failed in the original plan but were satisfied in the optimized one. For PTV, comparable $D_{97 \%}$ and its cold spot $D_{97}$ are also maintained in the optimized plan, but hot spots decrease, thereby indicating improved PTV coverage and dose homogeneity. Moreover, for OARs, most endpoints lower in the optimized process.

Averaged plan dosimetric endpoints for all evaluated 10 GYN IMRT cases are also investigated, as shown in Fig. 6. For PTV, the $D_{97 \%}$ and $V_{115 \%}$ are maintained, whereas the average $D_{99 \%}$ changes from 43.26Gy ( $\pm 0.41 \mathrm{~Gy})$ to $43.24 \mathrm{~Gy}( \pm 0.26 \mathrm{~Gy})$, and the $\mathrm{V}_{110 \%}$ decreased from $0.15 \%( \pm 0.18 \%)$ to $0.06 \%( \pm 0.10 \%)$, for the original and optimized plans, respectively. The average maximum dose of bowel, $\mathrm{V}_{45}$ of rectum and bladder, $\mathrm{V}_{30}$ of rectum and femoral heads, $\mathrm{V}_{20}$ and $\mathrm{V}_{10}$ of bone are decreased from $48.83 \mathrm{~Gy}( \pm 1.21 \mathrm{~Gy})$ to $48.72 \mathrm{~Gy}( \pm 1.00 \mathrm{~Gy}), 46.38 \%$ $( \pm 0.17 \%)$ to $43.81 \%( \pm 0.21 \%), 47.23 \%( \pm 0.11 \%)$ to $35.96 \%$ $( \pm 0.13 \%), 97.73 \%( \pm 0.02 \%)$ to $88.47 \%( \pm 0.11 \%), 14.53 \%$ $( \pm 0.10 \%)$ to $8.27 \%( \pm 0.05 \%), 72.05 \%( \pm 0.02 \%)$ to $59.02 \%$ $( \pm 0.04 \%)$, and $87.85 \%( \pm 0.01 \%)$ to $79.18 \%( \pm 0.01 \%)$, for the original and optimized plans, respectively. With slightly different trade-offs, the average maximum dose of rectum, bladder and femoral heads increase from 47.57Gy $( \pm 0.96 \mathrm{~Gy})$ to $49.4 \mathrm{~Gy}( \pm 1.29 \mathrm{~Gy}), 47.95 \mathrm{~Gy}( \pm 1.11 \mathrm{~Gy})$ to $48.42 \mathrm{~Gy}( \pm 1.18 \mathrm{~Gy})$, and $41.24 \mathrm{~Gy}( \pm 3.73 \mathrm{~Gy})$ to 41.52 Gy $( \pm 3.42 \mathrm{~Gy})$, respectively. However, these values effectively satisfy the corresponding constraints.

Results on difference significance analysis between the optimized plan and the original plan on all 10 cases are listed in Table 3. Among 13 observed endpoints, 6 are shown with significant differences between the original and optimized plans $(P<0.05)$, such as $D_{\text {max_rec }}, V_{30 \_r e c}$, $\mathrm{V}_{45 \_ \text {bla }}, \mathrm{V}_{10 \_ \text {bm }}, \mathrm{V}_{20 \_ \text {bm }}$, and $\mathrm{V}_{30 \_f h}$.

\section{Improvement tendency within optimization}

As mentioned above, four stages were designed in this multi-criteria optimization framework, namely, the first preparatory initial stage, the second violated constraint relaxing stage, the third backward relaxed constraint tightening stage, and the last constraint further tightening stage. Plan quality was inspected in the entire time

Table 2 Dosimetric endpoint comparison for case 2

\begin{tabular}{|c|c|c|c|c|}
\hline$\overline{\mathrm{ROI}}$ & Endpoints & Original plan & Optimal plan & Hard constraints \\
\hline PTV & $D_{99 \%}$ & $42.55 \mathrm{~Gy}$ & 42.96 Gy & 40.5 Gy \\
\hline PTV & $D_{97 \%}$ & $43.7 \mathrm{~Gy}$ & 43.7 Gy & 43.65 Gy \\
\hline PTV & $V_{115 \%}$ & 0 & 0 & 1 \\
\hline PTV & $V_{110 \%}$ & 0.22 & 0 & 10 \\
\hline Rectum & $D_{\max }$ & $47.7 \mathrm{~Gy}$ & 47.7 Gy & $51.75 \mathrm{~Gy}$ \\
\hline Bladder & $D_{\max }$ & $48.1 \mathrm{~Gy}$ & 46.5 Gy & $51.75 \mathrm{~Gy}$ \\
\hline Bowel & $D_{\max }$ & $49.3 \mathrm{~Gy}$ & 47.3 Gy & $51.75 \mathrm{~Gy}$ \\
\hline Femoral Heads & $D_{\max }$ & $44.9 \mathrm{~Gy}$ & 43.5 Gy & $51.75 \mathrm{~Gy}$ \\
\hline Bone & $V_{10}$ & $88.72 \%$ & $79.65 \%$ & $90 \%$ \\
\hline \multirow[t]{2}{*}{ Bone } & $V_{20}$ & $74.16 \%$ & $61.24 \%$ & $75 \%$ \\
\hline & & & & Soft constraints \\
\hline \multirow[t]{2}{*}{ Rectum } & $V_{30}$ & $99.97 \%$ & $96.40 \%$ & $60 \%$ \\
\hline & $V_{45}$ & $39.71 \%$ & $12.01 \%$ & $50 \%$ \\
\hline Bladder & $V_{45}$ & $44.64 \%$ & $13.13 \%$ & $50 \%$ \\
\hline Bowel & $V_{40}$ & $7.44 \%$ & $8.11 \%$ & $30 \%$ \\
\hline Femoral Heads & $V_{30}$ & $30.02 \%$ & $13.87 \%$ & $15 \%$ \\
\hline Bone & $V_{10}$ & $88.72 \%$ & $79.65 \%$ & $80 \%$ \\
\hline Bone & $V_{20}$ & $74.16 \%$ & $61.24 \%$ & $66 \%$ \\
\hline
\end{tabular}




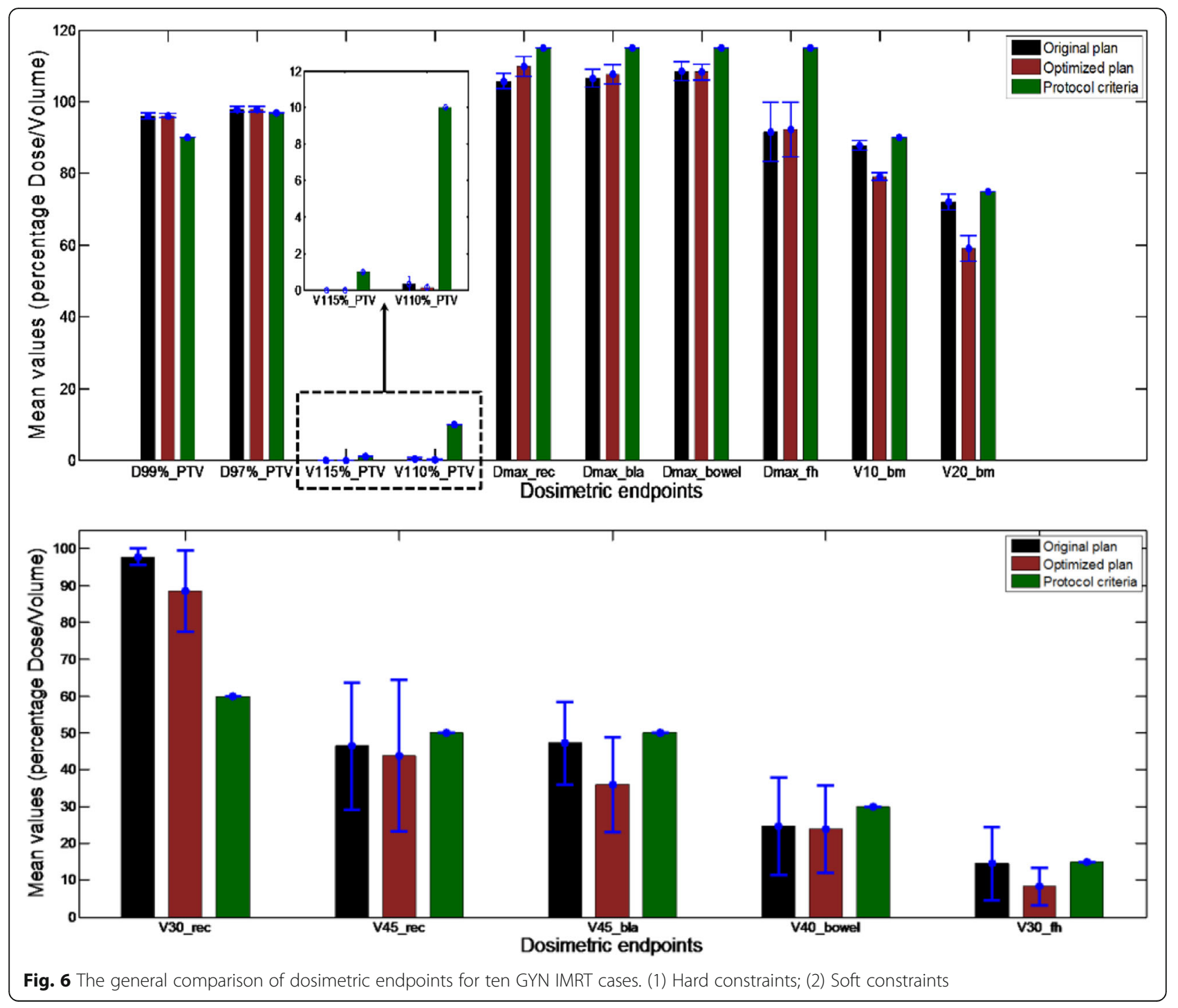

Table 3 Paired-T test of dosimetric endpoints

\begin{tabular}{lll}
\hline ROI & Endpoint & $P$ value \\
\hline PTV & $D_{99 \%}$ & 0.851042 \\
PTV & $V_{110 \%}$ & 0.184812 \\
Bladder & $D_{\max }$ & 0.234172 \\
Bowel & $D_{\max }$ & 0.787316 \\
Femoral heads & $D_{\max }$ & 0.563803 \\
Bowel & $V_{40}$ & 0.283576 \\
Rectum & $V_{45}$ & 0.496151 \\
Rectum & $D_{\max }$ & $\mathbf{0 . 0 0 0 4 5 9}$ \\
Rectum & $V_{30}$ & $\mathbf{0 . 0 1 0 8 1 5}$ \\
Bladder & $V_{45}$ & $\mathbf{0 . 0 0 4 4 1 6}$ \\
Bone & $V_{10}$ & $\mathbf{1 . 0 8 E}-\mathbf{0 8}$ \\
Bone & $V_{20}$ & $\mathbf{3 . 6 3 E}-\mathbf{0 6}$ \\
Femoral heads & $V_{30}$ & $\mathbf{0 . 0 0 5 6 4 1}$ \\
\hline Bold & &
\end{tabular}

Bold values indicate significant differences between the original and optimized plans $(P<0.05)$ with progression of the stages, and constraints changed during optimization.

Figure 7 shows an example of the clinical-relevant dostrimetric endpoint value changing process, with respect to its corresponding pre-defined constraints. The first stage ((a)-(b)) contains 30 voxel tuning iterations and no constraint adjustment. Normally, at the end of this stage, we cannot easily find a plan that meets all constraints, thereby resulting in the second stage, namely, relaxing violated constraints. This second stage begins at the violation of $\mathrm{V}_{40}$ bla and relaxing it from 65 to $65.5 \%$ and ends at the plan $\mathrm{V}_{40}$ bla was increased from 65.12 to $65.36 \%$ after optimization, requiring another 74 iterations for this case $((\mathrm{b})-(\mathrm{c})))$.In the third stage, we attempted to undo the constraint relaxations of stage 2 begins at 104th iterations. Between (c) and (d), the algorithm minimizes the dose-volume constraints for the bladder, because the dose-volume constraint for the bladder 


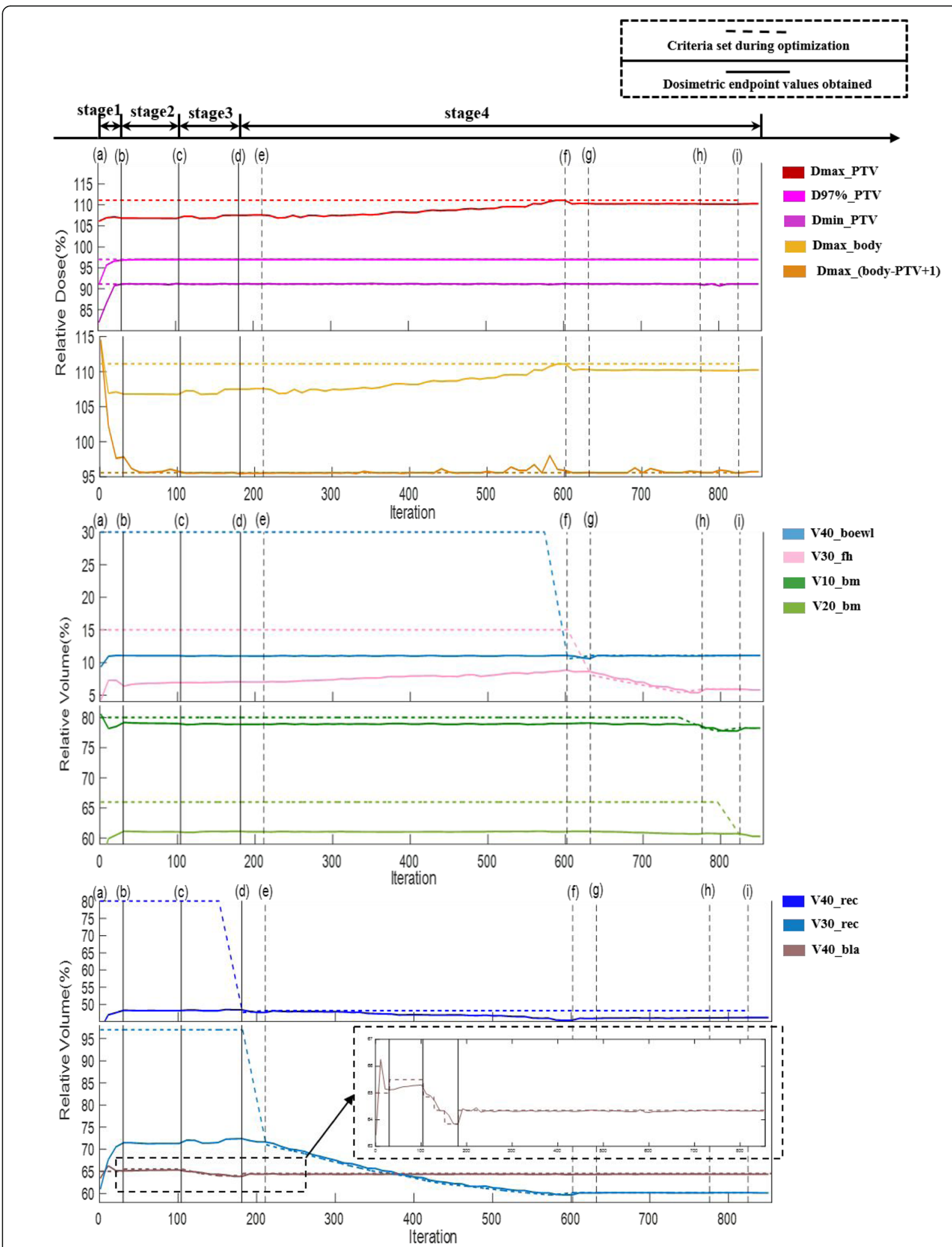

Fig. 7 Changing process of clinical relevant constraint and corresponding dosimetric endpoint value for cases 10. Dashed line: clinical relevant constraint; Solid line: corresponding dosimetric endpoint 
Table 4 Record on dosimetric endpoint value change with each stage ends for case 10

\begin{tabular}{|c|c|c|c|c|c|c|}
\hline $\mathrm{ROI}$ & Endpoint & Original plan & Stage 1 & Stage 2 & Stage 3 & Stage 4 \\
\hline PTV & $D_{99 \%}$ & $42.76 \mathrm{~Gy}$ & $42.92 \mathrm{~Gy}$ & $42.89 \mathrm{~Gy}$ & $42.91 \mathrm{~Gy}$ & $42.86 \mathrm{~Gy}$ \\
\hline PTV & $D_{97 \%}$ & $43.7 \mathrm{~Gy}$ & $43.7 \mathrm{~Gy}$ & 43.7 Gy & 43.7 Gy & 43.7 Gy \\
\hline PTV & $V_{115 \%}$ & 0 & 0 & 0 & 0 & 0 \\
\hline PTV & $V_{110 \%}$ & 0 & 0 & 0 & 0 & 0 \\
\hline Rectum & $D_{\max }$ & $46.1 \mathrm{~Gy}$ & $47.3 \mathrm{~Gy}$ & 47.5 Gy & 47.5 Gy & 49.7 Gy \\
\hline Bladder & $D_{\max }$ & $46.5 \mathrm{~Gy}$ & $47.5 \mathrm{~Gy}$ & $47.7 \mathrm{~Gy}$ & $47.9 \mathrm{~Gy}$ & $48.1 \mathrm{~Gy}$ \\
\hline Bowel & $D_{\max }$ & $46.5 \mathrm{~Gy}$ & $47.5 \mathrm{~Gy}$ & $47.7 \mathrm{~Gy}$ & $47.9 \mathrm{~Gy}$ & 47.7 Gy \\
\hline Femoral heads & $D_{\max }$ & 36.5 Gy & $38.9 \mathrm{~Gy}$ & 39.7 Gy & 39.9 Gy & 38.3 Gy \\
\hline Rectum & $V_{45}$ & $21.5 \%$ & $16.23 \%$ & $15.54 \%$ & $15.71 \%$ & $17.43 \%$ \\
\hline Bladder & $V_{45}$ & $45.78 \%$ & $30.07 \%$ & $29.47 \%$ & $30.01 \%$ & $32.28 \%$ \\
\hline Rectum & $V_{30}$ & $93.51 \%$ & $71.31 \%$ & $71.43 \%$ & $71.41 \%$ & $61.30 \%$ \\
\hline Bowel & $V_{40}$ & $11.25 \%$ & $11.07 \%$ & $10.88 \%$ & $10.95 \%$ & $10.98 \%$ \\
\hline Femoral heads & $V_{30}$ & $6.71 \%$ & $6.34 \%$ & $6.88 \%$ & $6.92 \%$ & $5.75 \%$ \\
\hline Bone & $V_{20}$ & $73.80 \%$ & $61.1 \%$ & $60.82 \%$ & $60.99 \%$ & $60.66 \%$ \\
\hline Bone & $V_{10}$ & $88.80 \%$ & $79.09 \%$ & $78.71 \%$ & $78.81 \%$ & $78.12 \%$ \\
\hline
\end{tabular}

is violated and relaxed previously. After optimization, the plan $\mathrm{V}_{40 \_ \text {bla }}$ was decreased from 65.36 to $64.34 \%$ at the end of stage 3. At stage 4, first, the $\mathrm{V}_{40}$ rec constraint is tightened, and until the algorithm failed to find a solution $((d)-(e))$. Then, the $V_{30 \_r e c}$ is considered. It undergoes a considerable reduction $((\mathrm{e})-(\mathrm{f}))$. Finally, the $\mathrm{V}_{40 \_ \text {bowel }}$ $((\mathrm{f})-(\mathrm{g})), \mathrm{V}_{30 \_f}((\mathrm{~g})-(\mathrm{h})), \mathrm{V}_{10 \_b m}((\mathrm{~h})-(\mathrm{i}))$ andV $\mathrm{V}_{20 \_b m}((\mathrm{i})-(\mathrm{j}))$ are tightened in turn. The entire optimization requires 855 iterations of voxel-based FMO optimization, which requires approximately $30 \mathrm{~min}$ to complete.

Table 4 and Fig. 8 illustrate an example of specific dosimetric endpoint values and its corresponding DVH curves at the end of each stage. Both of the dosimetric endpoint values and their corresponding DVH curves

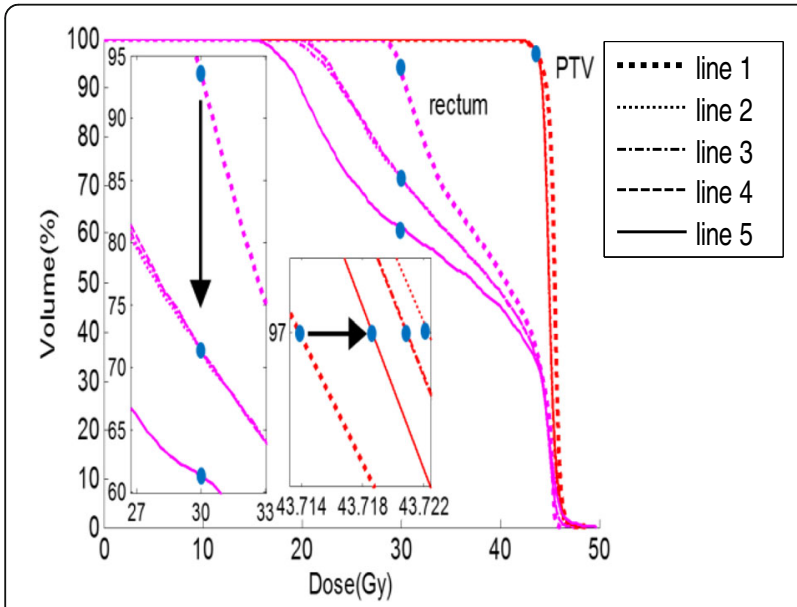

Fig. 8 Record on DVH change with each stage ends for case 10. Line 1: Original plan; line 2: Stage 1; line 3: Stage 2; line 4: Stage 3; line 5: Stage 4 gradually change as optimization proceeds. Relative drastic variations normally occur at the end of stages 1 and 4; for example, the $\mathrm{V}_{30}$ of rectum decreases to 71.31 and $61.30 \%$, respectively, compared with the original (93.51\%).

\section{Discussion}

For radiation therapy multi-criteria optimization, appropriate dosimetric goal setting and tuning methods are important. By considering these factors, we have successfully developed an automatic multi-criteria optimization framework with an automatic constraint adjustment strategy and a constraint-oriented voxel-based FMO method. The automatic multi-criteria optimization mechanism is similar to Breedveld's, but some changes were made, as follows. 1) Initial constraints are determined by experience rather than direct extraction from protocols, thereby providing an improved starting point for the optimization. 2) A more aggressive voxel tuning regime is used to accelerate the updating of the voxel weighting factor based on the difference from calculated dosimetric endpoint value to its criteria.

Ten clinical GYN cancer IMRT cases are used to evaluate the proposed system. The optimized plan not only improves the PTV dose homogeneity (with decreased PTV hotspots) but also provides a better OAR dose sparing for most cases. Among all the investigated dosimetric endpoints, most of them show significant improvement for the optimized plan $(P<0.05)$. Detailed improvement tendency within the optimization procedure is also studied, and every stage is imperative. The plan quality changes most in stages 1 and 4 . Although the $D_{\max }$ of rectum increases significantly, it still satisfied the 
protocol defined constraints, possibly because the $\mathrm{D}_{\max }$ of the rectum is set as a hard constraint and is thus not allowed to be adjusted during the entire optimization. Considering that the adjustment of the constraints and subsequent voxel-dependent parameter-based FMO are fully automatic, the proposed automatic multi-criteria optimization framework can dramatically decrease the current trial-and-error planning workload, thereby affording an efficient means to assure high plan quality consistency.

As depicted by the framework, a physician's clinical preference is paraphrased by the pre-defined constraint priority list. Thus, the final results embody their expected trade-offs. Consequently, the optimization result should be affected by the initial constraint value and their priorities. Our study trials show that constraints' ranking, especially for hard constraints, could dramatically affect the optimization results; even only one property change (i.e., priority) can influence optimization. For example, when the priority of the Dmax in body changes from priority 0 to 1 , the dose conformity changes dramatically and is far from the clinically accepted plan quality. For those ranked in soft constraints, by initial constraint value changes, the optimal DVH remains in shape. These factors could indicate the robustness of the proposed automatic multi-criteria optimization.

Furthermore, because the mathematical relationship between adjusting voxel-dependent parameters and satisfying DVH constraints remains unknown, the method we use to adjust voxel-dependent parameter is intuitive. Although physical dose sparing can be observed for the proposed optimized plan, the clinical benefit and the efficiency to plan generation in additional tumor sites should be further investigated and evaluated.

\section{Conclusions}

We have successfully developed an automatic multi-criteria optimization framework with an automatic constraint adjustment paradigm and a constraint-oriented voxel-based FMO method. The framework can dramatically reduce the current trial-and-error planning workload and afford an efficient method to assure high plan quality consistency.

\begin{abstract}
Abbreviations
BOXCQP: Bound constrained convex quadratic problems; CERR: Computational environment for radiotherapy research; DVC: Dose-volume-constraint; DVH: Dosevolume histogram; FMO: Fluence map optimization; GYN: Gynecology; IMRT: Intensity modulated radiation therapy; INTERTECC: International of Radiotherapy Technology Effectiveness in Cervical Cancer; LO: Lexicographic ordering; OAR: Organs-at-risk; PTV: Planning target volume; QIB: Quadrant infinite beam; ROI: Region-of-interest; TPS: Treatment planning system
\end{abstract}

\section{Acknowledgements}

The authors gratefully acknowledge the supported by National Key R\&D Program of China (NO.2017YFC0113203), National Natural Science Foundation of China (NO.81601577and 81571771), and Post-doctoral Science Foundation of China (NO.2016 M592510).

\section{Funding}

1) National Key R\&D Program of China (NO.2017YFC0113203);

2) National Natural Science Foundation of China (NO.81601577and 81571771);

3) Post-doctoral Science Foundation of China (NO.2016 M592510).

Availability of data and materials

Please contact author for data requests.

\section{Authors' contributions}

YM carried out the multi-criteria optimization studies and drafted the manuscript. FK participated in the multi-criteria optimization studies and performed the statistical analysis. YY provided experimental data and the clinical recommendations. LZ helped to conceive of the study and participated in experimental design. YL participated in experimental design and helped to draft the manuscript. TS conceived of the study, participated in experimental design and helped to draft the manuscript. All authors read and approved the final manuscript.

Ethics approval and consent to participate

Not applicable

Consent for publication

Not applicable

\section{Competing interests}

The authors declare that they have no competing interests.

\section{Publisher's Note}

Springer Nature remains neutral with regard to jurisdictional claims in published maps and institutional affiliations.

\section{Author details}

${ }^{1}$ Department of Biomedical Engineering, Southern Medical University, Guangzhou 510515, Guangdong, China. ²Department of Radiation Oncology, Sun Yat-Sen University Cancer Center,State Key Laboratory of Oncology in South China, Collaborative Innovation Center for Cancer Medicine, Guangzhou 510060, China. ${ }^{3}$ Department of Radiation Oncology, Zhejiang Cancer Hospital, Zhejiang 310022, Hangzhou, China.

Received: 23 March 2018 Accepted: 9 November 2018

Published online: 05 December 2018

\section{References}

1. Teh BS, Woo SY, Butler EB. Intensity modulated radiation therapy (IMRT): a new promising technology in radiation oncology. Oncologist. 1999;4(6):433-42.

2. Craft D. Multi-constraints optimization methods in radiation therapy planning: a review of technologies and directions. Am Soc Civil Eng. 2013; 157(11):1115-20.

3. Schlaefer A, Viulet T, Muacevic A, Fürweger C. Multiconstraints optimization of the spatial dose distribution. Med Phys. 2013;40(12):1720-9.

4. Fiege J, Mccurdy B, Potrebko P, Champion H, Cull A. PARETO: a novel evolutionary optimization approach to multiobjective IMRT planning. Med Phys. 2011;38(9):5217-29.

5. Craft D, Halabi T, Bortfeld T. Exploration of tradeoffs in intensity-modulated radiotherapy. Phys Med Biol. 2005;50(24):5857-68.

6. Müller BS, Shih HA, Efstathiou JA, Bortfeld T, Craft D. Multiconstraints plan optimization in the hands of physicians: a pilot study in prostate cancer and brain tumors. Radiat Oncol. 2017;12(1):168-78.

7. Potrebko PS, Fiege J, Biagioli M, Poleszczuk J. Investigating multi-objective fluence and beam orientation IMRT optimization. Phys Med Biol. 2017; 62(13):5228-44.

8. Wahl N, Bangert M, Kamerling CP, Ziegenhein P, Bol GH, Raaymakers BW, et al. Physically constrained voxel-based penalty adaptation for ultra-fast IMRT planning. J Appl Clin Med Phys. 2016;17(4):172-89.

9. Williams MJM, Bailey M, Forstner D, Metcalfe P. Multicentre quality assurance of intensity-modulated radiation therapy plans: a precursor to clinical trials. J Med Imaging Radiat Oncol. 2007;51(5):472-9.

10. Chung HT, Lee B, Park E, Lu JJ, Xia P. Can all centers plan intensity-modulated radiotherapy (IMRT) effectively? An external audit of dosimetric comparisons between three-dimensional conformal radiotherapy and IMRT for adjuvant 
chemoradiation for gastric cancer. Int J Radiat Oncol Biol Phys. 2008;71(4): 1167-74.

11. Das I, Cheng C-W, L Chopra K, Mitra R, Srivastava S, Glatstein E. Response: re: intensity-modulated radiation therapy dose prescription, recording, and delivery: patterns of variability among institutions and treatment planning systems. J Natl Cancer Inst. 2008;100(17):1265-7.

12. Wilkens JJ, Alaly JR, Zakarian K, Thorstad WL, Deasy JO. IMRT treatment planning based on prioritizing prescription goals. Phys Med Biol. 2007;52(6): 1675-92.

13. Jee KW, McShan DL, Fraass BA. Lexicographic ordering: intuitive multiconstraints optimization for IMRT. Phys Med Biol. 2007;52(7):1845-61.

14. Breedveld S, Storchi PRM, Keijzer M, et al. A novel approach to multi-criteria inverse planning for IMRT. Phys Med Biol. 2007;52(20):6339.

15. Breedveld S, Storchi PRM, Heijmen BJM. The equivalence of multi-criteria methods for radiotherapy plan optimization. Phys Med Biol. 2009:54(23):7199.

16. Breedveld S, Storchi PRM, Voet PWJ, et al. iCycle: integrated, multicriterial beam angle, and profile optimization for generation of coplanar and noncoplanar IMRT plans. Med Phys. 2012;39(2):951-63.

17. Holdsworth C, Kim M, Liao J, Phillips M. The use of a multiobjective evolutionary algorithm to increase flexibility in the search for better IMRT plans. Med Phys. 2012;39(4):2261-74.

18. Zarepisheh M, Uribesanchez AF, Li N, Jia X, Jiang SB. A multiconstraints framework with voxel-dependent parameters for radiotherapy treatment plan optimization. Med Phys. 2014;41(4):041705.

19. Cotrutz C, Xing L. Using voxel-dependent importance factors for interactive DVH-based dose optimization. Phys Med Biol. 2002;47(10):1659-69.

20. Cotrutz C, Xing L. IMRT dose shaping with regionally variable penalty scheme. Med Phys. 2003;30(4):544-51.

21. Wu C, Olivera GH, Jeraj R, Keller H, Mackie TR. Treatment plan modification using voxel-based weighting factors/dose prescription. Phys Med Biol. 2003; 48(15):2479-91.

22. Breedveld S, Storchi PR, Keijzer M, Heijmen BJ. Fast, multiple optimizations of quadratic dose objective functions in IMRT. Phys Med Biol. 2006:51(14): 3569-79.

23. Kalinin $E D$, J. A method for fast 3-D IMRT dose calculations the quadrant infinite beam (QIB) algorithm. 45th annual meeting of the American Association of Physicist in medicine. 2003.

24. Anders A, Mikael S, Avo T. A pencil beam model for photon dose calculation. Med Phys. 1992;19(2):263-73.

25. Deasy JO, Blanco Al, Clark VH. CERR: a computational environment for radiotherapy research. Med Phys. 2003;30(5):979-85.

26. Voglis C, Lagaris IE. BOXCQP: an algorithm for bound constrained convex quadratic problems. IC-SCCE. 2004.

27. Bortfeld T, Stein J, Preiser K. Clinically relevant intensity modulation optimization using physical constraints. In: Proceeding of XII international conference on the use of computers in radiationtherapy. Salt Lake City, Utah; 1997. p. 1-4.

Ready to submit your research? Choose BMC and benefit from:

- fast, convenient online submission

- thorough peer review by experienced researchers in your field

- rapid publication on acceptance

- support for research data, including large and complex data types

- gold Open Access which fosters wider collaboration and increased citations

- maximum visibility for your research: over $100 \mathrm{M}$ website views per year

At $\mathrm{BMC}$, research is always in progress.

Learn more biomedcentral.com/submissions 